EXTENDED REPORT

\title{
Tenascin and aggrecan expression by articular chondrocytes is influenced by interleukin 1 $\beta$ : a possible explanation for the changes in matrix synthesis during osteoarthritis
}

\author{
D Pfander, N Heinz, P Rothe, H-D Carl, B Swoboda
}

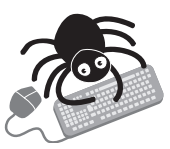

Web extra figure $\mathrm{W} 1$ can be seen on the website at http://www.annrheumdis. com/supplemental

See end of article for authors' affiliations

.....................

Correspondence to: Dr D Pfander, Division of Orthopaedic

Rheumatology,

Department of

Orthopaedic Surgery im

Waldkrankenhaus St

Marien, University of

Erlangen, Nuremberg,

Rathsbergerstr 57 ,

D-91054 Erlangen,

Germany;

dpfander@t-online.de

Accepted 4 June 2003

\begin{abstract}
Objective: To analyse the distribution patterns of tenascin and proteoglycans in normal and osteoarthritic cartilage, and to determine the effect of interleukin $1 \beta$ (ILI $\beta$ ) on aggrecan and tenascin expression by human articular chondrocytes in vitro.

Methods: Normal and osteoarthritic cartilage and bone samples were obtained during total knee replacements or necropsies. After fixation and decalcification, paraffin embedded specimens were sectioned perpendicular to the surface. Specimens were graded according to Mankin and subdivided into those with normal, and mild, moderate, and severe osteoarthritic lesions. Serial sections were immunostained for tenascin. Tenascin expression by healthy and osteoarthritic chondrocytes was quantified by real time polymerase chain reaction (PCR). Furthermore, in cell culture experiments, human articular chondrocytes were treated with 0.1 or $10 \mathrm{ng} / \mathrm{ml} \mathrm{IL1} \beta$. Real time PCR analyses of aggrecan and tenascin transcripts (normalised 18S rRNA) were conducted to determine the effect of ILI $\beta$ on later mRNA levels.

Results: Tenascin was immunodetected in normal and osteoarthritic cartilage. In osteoarthritic cartilage increased tenascin staining was found. Tenascin was found specifically in upper OA cartilage showing a strong reduction of proteoglycans. Greatly increased tenascin transcript levels were detected in osteoarthritic cartilage compared with healthy articular cartilage. IL1 $\beta$ treatment of articular chondrocytes in vitro significantly increased tenascin transcripts ( $200 \%$ of control) and strongly reduced aggrecan mRNA levels ( $\sim 42 \%$ of control).

Conclusions: During progression of osteoarthritis the switch in matrix synthesis occurs mainly in upper osteoarthritic cartilage. Furthermore, changes in synthesis patterns of osteoarthritic chondrocytes may be significantly influenced by IL $\beta$, probably diffusing from the joint cavity within the upper osteoarthritic cartilage.
\end{abstract}

O steoarthritis (OA) a degenerative disease of articular cartilage is accompanied by typical changes of the periarticular bone (osteophytes, sclerosis) and inflammatory reactions of the synovial membrane. 'Specifically, fibrillations and fissuring of the surface, progressive loss of proteoglycans, formation of chondrocyte clusters, and in late stage OA decreased cell density are signs of the progressive damage of hyaline cartilage. It has been repeatedly shown that disease progression depends on the severity of the cartilage damage. Therefore, matrix synthesis by articular chondrocytes is a critical factor in the maintenance of cartilage integrity in healthy joints. Many efforts have been devoted to characterising the different matrix compositions under normal and pathological conditions such as OA. ${ }^{2-5}$ Evidence has shown that articular chondrocytes change their synthesis pattern, resulting in an insufficient matrix unable to absorb and respond to mechanical load. ${ }^{6}$

In our previous studies we showed an increased deposition of tenascin, collagen type I, and fibronectin in upper cartilage during experimental $\mathrm{OA}$ in rabbits. ${ }^{7}$ Tenascin is a large glycoprotein composed of six subunits. Each tenascin subunit consists of an epidermal growth factor-like repeat, a fibronectin (Fn) type III repeat, and a fibrinogen sequence. ${ }^{8}$ On the other hand, it has been shown that distinct matrix proteins-for example thrombospondin and aggrecan, are decreased in OA, and it is reasonable to assume that the unique biomechanical properties of OA cartilage are weakened. ${ }^{9}{ }^{10}$ Furthermore, it has been shown that proinflammatory cytokines such as interleukin $1 \beta$ (ILI $\beta$ ), tumour necrosis factor $\alpha$ and others are secreted by synoviocytes into the joint cavity. ${ }^{11}{ }^{12}$ In healthy joints ILl $\beta$ is counteracted by ILl receptor antagonist, whereas in OA joints this balance is disturbed and ILI $\beta$ initiates different catabolic pathways by activating the transcription factor $\mathrm{NF \kappa B}$ and several signal cascades. ${ }^{13-15}$

Beside its effects on apoptosis, inducible nitric oxide synthase expression, and matrix metalloproteinases expression, ILl $\beta$ has significant effects on matrix molecule expression by articular chondrocytes (for review see MartelPelletier $e a^{15}$ ). ILl $\beta$ decreases type II collagen and aggrecan expression. ${ }^{16}{ }^{17}$ As mentioned above osteoarthritic chondrocytes are considered to change their synthesis pattern, thereby down regulating aggrecan expression and enhancing the expression of other matrix molecules. ${ }^{2-6} 1819$ This phenomenon could be explained by intrinsic mechanisms such as differentiation or de-differentiation processes of chondro-

\footnotetext{
Abbreviations: HBSS, Hanks's buffered salt solution; IL, interleukin; OA, osteoarthritis; PBS, phosphate buffered saline; PCR, polymerase chain reaction
} 
$\mathbf{A}$

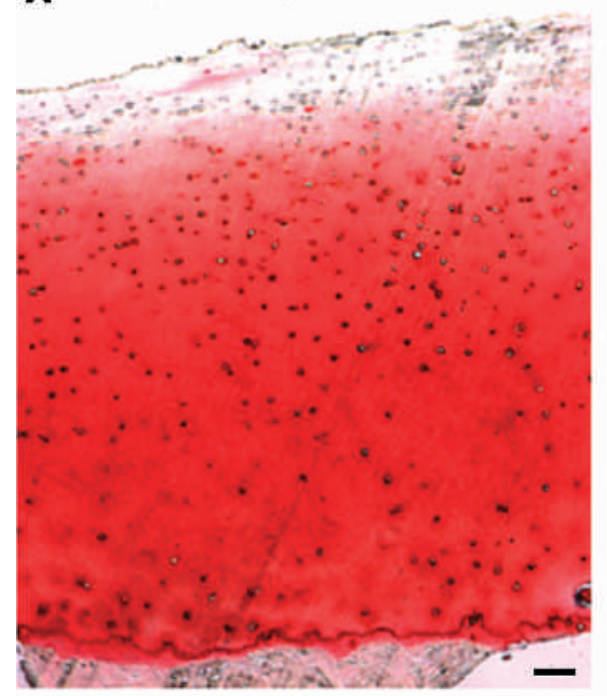

C

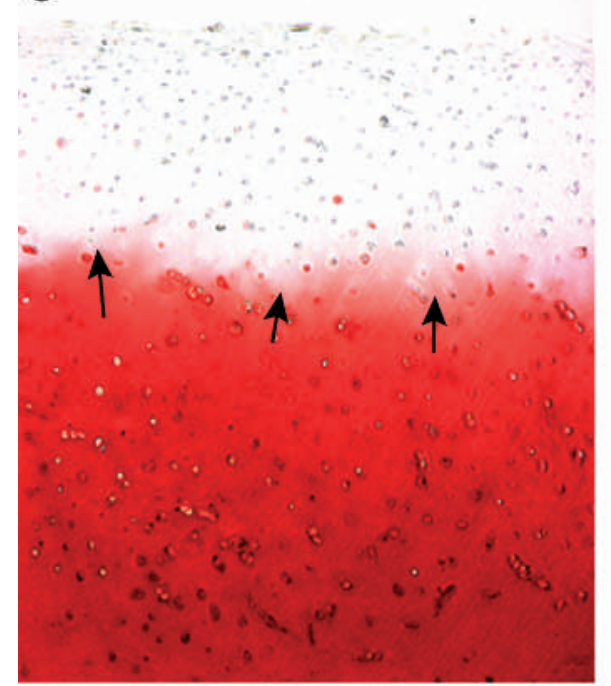

E

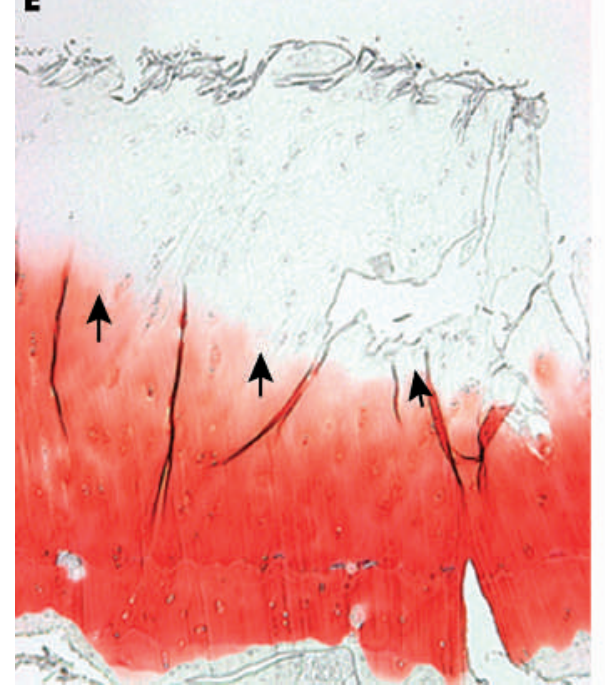

B

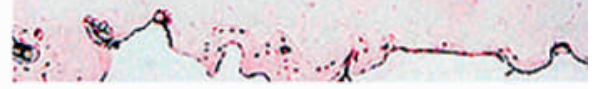

D

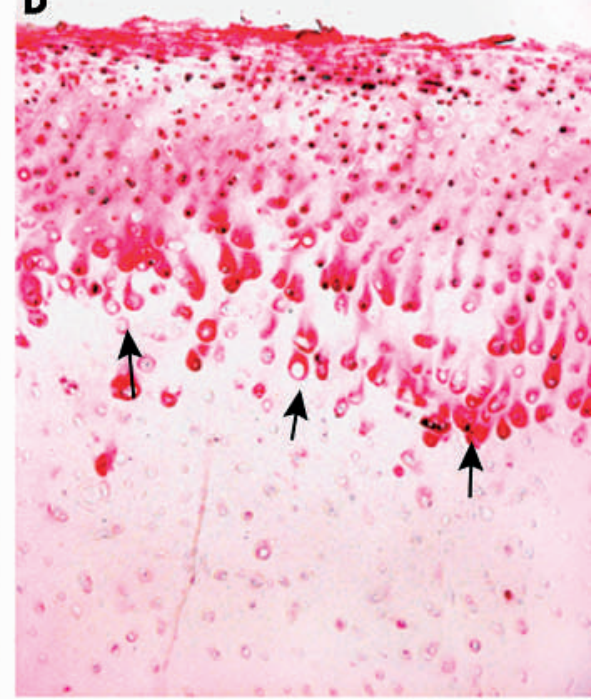

$\mathbf{F}$

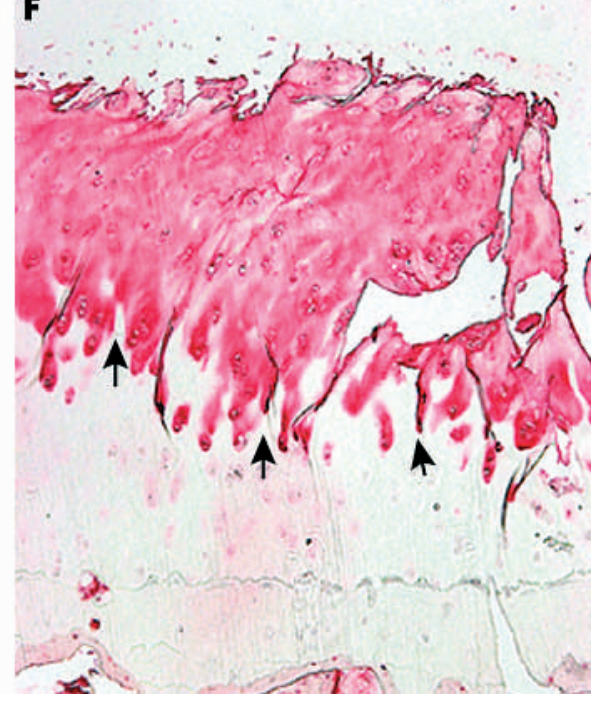

Figure 1 Serial sections of normal and OA cartilage. The tenascin deposition in healthy cartilage varied interindividually. Normal cartilage stained for proteoglycans (A) and immunostained for tenascin (B) shows pericellular/territorial tenascin deposition around the superficial and upper middle zone chondrocytes (weak immunostaining). (C) Mild OA lesion with decreased proteoglycan staining of the upper cartilage (safranin $O$ staining). (D) Immunohistochemical analysis of tenascin in the same section as shown in C (note the territorial staining around the middle zone chondrocytes indicated by arrows). (E) Severe OA cartilage lesion showing a strong reduction of proteoglycans, deep clefts within the deep zone, and chondrocyte clusters in all zones (safranin $O$ staining). (F) Immunostaining of tenascin in the same section as shown in $E$ (note the strong tenascin staining in cartilage areas showing the absence of proteoglycans indicated by arrows). The bar in A represents $100 \mu \mathrm{m}$ in A-F. 
Table 1 Sequences of primers and probes

\begin{tabular}{llll}
\hline & Forward & Reverse & Probe \\
\hline Tenascin & ATCCGGACCAAAACCATCAG & AATGTCGGAAATGGTTAGGTITTC & CGACAGAGGCCCTGCCCCTC \\
Aggrecan & ACTTCCGCTGGTCAGATGGA & TCTCGTGCCAGATCATCACC & CCATGCAATTGAGAACTGGCGCC \\
\hline
\end{tabular}

cytes, as well as by extrinsic factors, for example, proinflammatory cytokines or mechanical load. ${ }^{20}$

Two previous studies have shown that tenascin is present in human normal and OA cartilage. ${ }^{21}{ }^{22}$ We have additionally demonstrated that beside tenascin other matrix components such as fibronectin and type I collagen are deposited within the upper OA cartilage of rabbits. In view of these observations, we suggested that a common mediator controlled pathway may induce these changes in matrix synthesis by OA chondrocytes. ${ }^{7}$ However, it is still unclear whether ILl $\beta$ is responsible for the changes in matrix gene expression which probably weaken the unique biomechanical properties of articular cartilage. To test this hypothesis we determined the presence and distribution of tenascin compared with proteoglycans in normal and OA cartilage. Furthermore, cell culture experiments with human articular chondrocytes, cultured in the absence and presence of ILl $\beta$, were conducted. The effect of ILl $\beta$ on aggrecan and tenascin mRNA expression levels was determined by real time polymerase chain reaction (PCR).

\section{MATERIALS AND METHODS}

\section{Patients and samples}

Cartilage and bone samples were obtained from patients who had been admitted to hospital in the Division of Orthopaedic Rheumatology, University Erlangen-Nuremberg, Germany. The following clinical data had been obtained: age, sex, patient history, physical examination, haematological analysis (complete blood count, rheumatoid factors, erythrocyte sedimentation rate, serum electrophoresis, $\mathrm{C}$ reactive protein, and other serum diagnostic tests), and $x$ ray examinations of the respective knee joints carried out. Data were carefully reviewed to exclude any secondary forms of $\mathrm{OA}$ and rheumatoid arthritis. Thirty one human cartilage samples were obtained from 29 patients undergoing total knee replacement. Nine human normal cartilage samples were obtained from five donors during necropsies and from one patient undergoing a reconstruction of the anterior cruciate ligament. The localisation of the excised samples was carefully documented by videoprints. The specimens were immediately fixed in $4 \%$ paraformaldehyde in phosphate buffered saline (PBS), decalcified in diethyl-pyrocarbonate treated 0.2 M EDTA, embedded in paraffin wax, and then cut into $6 \mu \mathrm{m}$ thick sections perpendicular to the surface. Serial sections were stained with safranin $\mathrm{O}$ and used for immunohistochemistry.

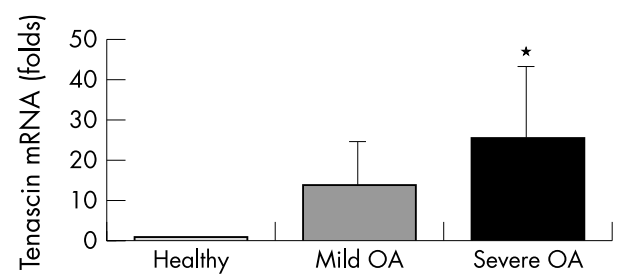

Figure 2 Bars represent the several-fold changes of tenascin mRNA levels (normalised to 18S rRNA) in mild and severe OA lesions compared with healthy cartilage samples (one fold expression). Bars are means (SD). ${ }^{*} p<0.05$ versus healthy samples.
Histological-histochemical grading

Sections of normal and OA cartilage specimens routinely stained with safranin $\mathrm{O}$ were graded according to Mankin et al by two different observers. ${ }^{23}$ The samples were classified as normal as well as mild, moderate, and severe OA lesions as described previously ${ }^{9}$ : Mankin score $0-1$, normal cartilage (9 samples); 2-5, mild OA lesion (8 samples); 6-9, moderate OA lesion (12 samples); $\geqslant 10$, severe OA lesion (11 samples).

\section{Immunohistochemistry}

After deparaffinisation, the sections were incubated with $2 \mathrm{mg} / \mathrm{ml}$ hyaluronidase (Merck, Darmstadt, Germany) in PBS ( $\mathrm{pH}$ 5.5) for 15 minutes, followed by digestion with $1 \mathrm{mg} / \mathrm{ml}$ pronase in PBS ( $\mathrm{pH} 7.5$ ) for 30 minutes (Boehringer, Mannheim, Germany). Non-specific binding of the antibodies was blocked with $5 \%$ bovine serum albumin in PBS. Each step was followed by extensive washing in PBS. Sections were then incubated overnight at $4^{\circ} \mathrm{C}$, with a human tenascin-specific monoclonal antibody (Sigma, Munich, Germany) diluted 1:1000. Negative control cartilage sections were incubated with mouse control isotypes. After incubation with the first antibody each step was followed by extensive washing in Tris buffered saline. This was then followed by incubation with a biotinylated antimouse IgG from rabbits, diluted 1:500. Then, a complex of streptavidin and biotin labelled with alkaline phosphatase was added according to the manufacturer's protocol (Dako, Hamburg, Germany). Finally the sections were coloured with Fast Red (Sigma, Munich, Germany).

\section{Sample acquisition and RNA preparation}

Eight samples of OA cartilage were obtained from eight patients who had undergone total knee replacement. Additionally, three normal samples were obtained at necropsy from patients who had had no known history of joint disease. The cartilage areas for RNA isolation were macroscopically graded as four mild (softening, fibrillations) and four severe (softening, yellowish colour, fissuring down to the bone or calcified cartilage) OA lesions and documented by videoprints. Cartilage samples were rapidly dissected and stored until preparation at $-70^{\circ} \mathrm{C}$. The frozen tissue was shredded under liquid nitrogen and total RNA was isolated from cartilage with the Nucleo-Spin-RNA-II-Kit (Clontech

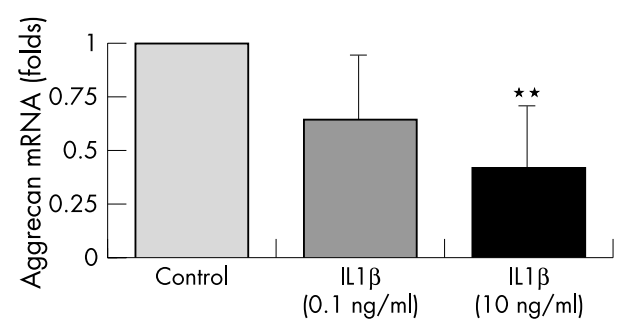

Figure 3 Bars represent the several-fold changes of aggrecan mRNA levels (normalised to 18S rRNA) of IL1 $\beta$ treated chondrocytes compared with untreated control chondrocytes (one fold expression). Articular chondrocytes were cultured under serum free conditions for 18 hours in the absence or presence of 0.1 and $10 \mathrm{ng} / \mathrm{ml} \mathrm{IL1} \beta$, respectively. Results are from four independent cell culture experiments. Bars represent means (SD). ${ }^{* *} p<0.01$ versus control. 


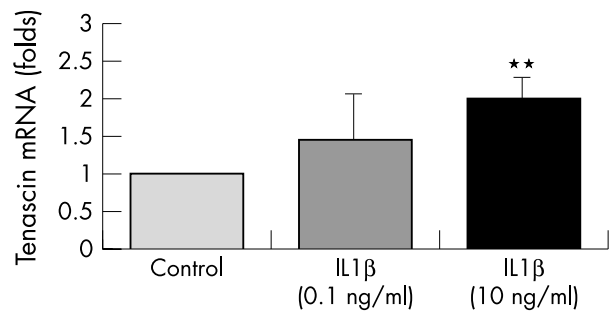

Figure 4 Bars represent the several-fold changes of tenascin mRNA levels (normalised to 18S rRNA) of IL1 $\beta$ treated chondrocytes compared with untreated control chondrocytes (one fold expression). Articular chondrocytes were cultured under serum free conditions for 18 hours in the absence or presence of 0.1 and $10 \mathrm{ng} / \mathrm{ml}$ IL $1 \beta$, respectively. Results are from four independent experiments. Bars represent means (SD). ${ }^{* *} p<0.01$ versus control.

Laboratories, Palo Alto, USA) as recommended by the manufacturer. Total RNA yields were calculated by measurement of the extinction at $260 \mathrm{~nm}$ (UltrospecIII, LKB).

\section{Reverse transcription and real time PCR}

After isolation, RNA was transcribed into single complementary DNA using AMV reverse transcriptase (Boehringer, Mannheim, Germany) and random hexamer primers according to the manufacturer's instructions.

For real time PCR analyses cDNAs from different cartilage samples or four independent cell culture experiments were diluted to a final concentration of $10 \mathrm{ng} / \mu \mathrm{l}$. For PCR reactions TaqMan-Universal Mastermix (Applied Biosytems) was used. 50 ng cDNA was used as template to determine the relative amount of mRNA by real time PCR (ABI Prism 7700 sequence detection system) using specific primers and probes (table 1) (Qiagen Operon, Cologne, Germany). The reaction was conducted as follows: $95^{\circ} \mathrm{C}$ for 4 minutes, and 40 cycles of 15 seconds at $95^{\circ} \mathrm{C}$ and 1 minute at $60^{\circ} \mathrm{C}$. $18 \mathrm{~S}$ rRNA was amplified as an internal control. Cycle threshold values were measured and calculated by the sequence detector software. Relative amounts of mRNA were normalised to 18S rRNA (Applied Biosystems) and calculated with the software program Microsoft Excel. Relative mRNA contents were calculated as $\mathrm{x}=2^{-\Delta \Delta \mathrm{Ct}}$, in which $\Delta \Delta \mathrm{Ct}=\Delta \mathrm{E}-\Delta \mathrm{C}$ and $\Delta \mathrm{E}=\mathrm{Ct}_{\text {sample }}-\mathrm{Ct}_{18 \mathrm{~s}}$ and $\Delta \mathrm{C}=\mathrm{Ct}_{\text {control/normal }}-\mathrm{Ct}_{18 \mathrm{~s}}$. For statistical analyses the Mann-Whitney $\mathrm{U}$ test was used.

\section{Chondrocyte culture}

Human articular chondrocytes were isolated from cartilage obtained during knee replacements with two digestion steps. Firstly, articular cartilage was digested in Hanks's buffered salt solution (HBSS) containing pronase $2 \mathrm{mg} / \mathrm{ml}$ (30 minutes at $37^{\circ} \mathrm{C}$ ) followed by a collagenase $180 \mathrm{U} / \mathrm{ml}$ in HBSS $\left(6\right.$ hours at $37^{\circ} \mathrm{C}$ ). After quantification of isolated chondrocytes with a haemocytometer, two million chondrocytes were plated onto $5 \mathrm{~cm}$ culture dishes in Dulbecco's modified Eagle's medium containing $10 \%$ bovine fetal serum, $2 \mathrm{mM}$ glutamine, and $50 \mathrm{U} / \mathrm{ml}$ streptomycin/penicillin. Chondrocytes were cultured until they reached confluency (day 7 after plating). Cells were then treated with either 0.1 or $10 \mathrm{ng} / \mathrm{ml}$ ILl $\beta$ under serum free conditions. After 18 hours, total RNA was harvested with the Nucleo-SpinRNA-II-Kit (Clontech Laboratories, Palo Alto, USA) as recommended by the manufacturer.

\section{RESULTS}

In the first set of our experiments we analysed the tenascin distribution in human normal and OA cartilage samples. In normal cartilage showing a smooth surface and homogeneous proteoglycan staining, tenascin was present in the pericellular territorial matrix of the superficial and middle zone chondrocytes and on the surface lining. In a few specimens a slight interterritorial matrix staining in the deep zone was even seen. Further, osteocytes and osteoblasts of the subchondral bone showed tenascin expression. The intensity of the tenascin staining in healthy cartilage varied between specimens from different donors (figs $1 \mathrm{~A}$ and $\mathrm{B}$ and figs WIA and B (available at http://www.annrheumdis.com/ supplemental)). Control slides incubated with control isotypes showed no staining (not shown).

In mild and moderate OA lesions decreased proteoglycan staining next to the cartilage surface was accompanied by increased territorial and interterritorial tenascin deposition. In addition, we detected the highest tenascin depositions specifically in those cartilage areas showing the greatest reduction of safranin O staining (figs $\mathrm{IC}$ and D and figs WIE and F (http://www.annrheumdis.com/supplemental)). In the middle and upper deep zones some specimens showed a territorial tenascin deposition (figs $\mathrm{IC}$ and D). Pannus-like tissue covering OA cartilage showed a very strong immunostaining for tenascin (figs WIC and D (http://www. annrheumdis.com/supplemental)). Even in the surrounding matrix of chondrocyte clusters a strong tenascin staining was determined.

In OA specimens with high Mankin's grade (severe OA lesion) strongly increased immunostaining of tenascin was found (figs $\mathrm{IE}$ and F). As described previously, the highest tenascin depositions were immunodetected specifically in upper OA cartilage characterised by great reduction of proteoglycans (figs WlG and $\mathrm{H}$ (http://www.annrheumdis. com/supplemental)). Significantly higher transcript levels of tenascin were detected in severe OA cartilage lesions than in healthy articular cartilage by real time PCR (normalised to 18S rRNA) (fig 2).

In a previous study we detected increased protein levels of tenascin, fibronectin, and type I collagen next to the surface of rabbit OA cartilage. ${ }^{7}$ Thus we suggested that a common mediator controlled pathway is responsible for the changes in chondrocytic synthesis pattern next to the joint space. In addition, Aigner et al showed that OA chondrocytes next to the cartilage surface reduce the expression of aggrecan. ${ }^{10}$ On the other hand, we and other groups have demonstrated that tenascin and other molecules are up regulated in OA. To test the hypothesis that ILl $\beta$ one of the most prominent mediators in OA, diffusing into upper OA cartilage, is responsible for at least some of the changes in expression pattern, cell culture experiments were conducted. Therefore OA chondrocytes were isolated and cultured in monolayer. After the cells reached confluency, chondrocytes were treated with two different concentrations of IL1 $\beta$ under serum free conditions. After 18 hours total RNA was harvested and aggrecan and tenascin transcripts were quantified by real time PCR (normalised to $18 \mathrm{~S}$ rRNA). Figures 3 and 4 show the results of four culture series. As shown, we determined a strong stimulation of tenascin mRNA expression ( $\sim 200 \%$ of control) by ILl $\beta$, whereas the aggrecan transcript levels $(\sim 42 \%$ of control) were significantly reduced, further supporting the idea that IL1 $\beta$ is responsible for changes in matrix synthesis during OA.

\section{DISCUSSION}

In this study we have shown that changes of matrix gene expression in OA cartilage are strongly influenced by ILl $\beta$. Firstly, we analysed the protein expression pattern of tenascin in healthy and OA cartilage. In healthy articular cartilage different staining intensities of tenascin were seen in the superficial zone and in the upper middle zone, mainly in the territorial matrix of the articular chondrocytes, confirming the results of Salter. ${ }^{21}$ In pannus-like tissue 
covering the surface of OA cartilage, strong tenascin staining was detected. In moderate and severe OA lesions increased territorial and interterritorial immunostaining was determined, mainly in cartilage areas showing a great reduction of safranin O staining. Our previous observations on rabbit OA cartilage and these extended findings further strengthen the idea of a common mediator controlled pathway responsible for the observed changes in cartilage matrix composition. Several groups have provided evidence that ILl $\beta$ is one of the most dominant cytokines in OA, stimulating different catabolic pathways by activating the transcription factor $\mathrm{NF \kappa B}$ and several other signal cascades as well. ${ }^{15}$ Interestingly, it has been repeatedly shown that ILl $\beta$ inhibits aggrecan expression and stimulates expression of matrix metalloproteinases and aggrecanase, thereby contributing to the pathogenesis of OA. ${ }^{15}$ Furthermore, ILl $\beta$ has been shown to accumulate in upper OA cartilage, thus eventually influencing the synthesis pattern of articular chondrocytes. ${ }^{13}$ A previous study on stromal cells has demonstrated that ILl stimulates the expression of tenascin on RNA, and the protein level. ${ }^{24}$ Taken together, these findings suggest that IL1 $\beta$ could be a key mediator in the regulation of chondrocytic synthesis pattern. To prove the hypothesis that chondrocytic matrix molecule expression is modulated by IL1 $\beta$, cell culture experiments were conducted. Consistent with this notion, real time PCR analyses disclosed that the action of ILI $\beta$ significantly reduced aggrecan mRNA levels. Beside its inhibiting effects on aggrecan expression, tenascin message was significantly stimulated by addition of IL1 $\beta$ to the chondrocyte cultures. Our findings are in accordance with a previous study, which demonstrated that ILl $\beta$ enhances the immunohistological staining of tenascin in cartilage explants. ${ }^{25}$ In addition, we and other groups found significantly increased tenascin transcripts in OA cartilage, further indicating the significance of changes in chondrocytic expression patterns in vivo. ${ }^{26}$ However, we cannot rule out the possibility that chondrocytic expression patterns are influenced by other important mechanisms, such as mechanical load, oxygenation levels, growth factors, and differentiation processes. ${ }^{27}{ }^{28}$ None the less, our findings and those of others suggest a crucial role for ILl $\beta$ in the observed switch of matrix gene expression during disease progression, thereby contributing to the cartilage changes seen in OA.

\section{ACKNOWLEDGEMENTS}

We thank Maria Gesslein for her skilful technical assistance. This work was supported in part by the Deutsche Forschungsgemeinschaft ( $\mathrm{PF} 383 / 1-1$ ), the BMBF, and the Interdisciplinary Centre for Clinical Research at the University Hospital Erlangen-Nuremberg (project D5).

\section{Authors' affiliations}

D Pfander, N Heinz, P Rothe, H-D Carl, B Swoboda, Division of Orthopaedic Rheumatology, Department of Orthopaedic Surgery, University of Erlangen-Nuremberg, Erlangen, Germany

\section{REFERENCES}

1 Kuettner KE, Goldberg V. Osteoarthritis disorders. Rosemont, American Academy of Orthopaedic Surgeons Symposium Series 1995:27-45.

2 Nimni M, Deshmukh K. Differences in collagen metabolism between normal and osteoarthritic human articular cartilage. Science 1973;181:751-2.

3 Gay S, Rhodes RK. Immunohistological demonstration of distinct collagens in normal and osteoarthritic joints. Semin Arthritis Rheum 1981;11:43-4.
4 Jones KL, Brown M, Ali SY, Brown RA. An immunohistochemical study of fibronectin in human osteoarthritic and disease free articular cartilage. Ann Rheum Dis 1987;46:809-15.

5 Pullig O, Weseloh G, Gaver S, Swoboda B. Osteopontin is expressed by adult human osteoarthritic chondrocytes: protein and mRNA analysis of normal and osteoarthritic cartilage. Matrix Biol 2000;19:245-55.

6 Aigner T, Bertling W, Stöss H, Weseloh G, von der Mark K. Independent expression of fibril-forming collagens I, II and III in chondrocytes of human osteoarthritic cartilage. J Clin Invest 1993:91:829-37.

7 Pfander D, Rahmanzadeh R, Scheller EE. Presence and distribution of collagen II, Collagen I, fibronectin and tenascin in rabbit normal and osteoarthritic cartilage. J Rheumatol 1999;26:386-94.

8 Weller A, Beck S, Ekholm P. Amino acid sequence of mouse tenascin and differential expression of two tenascin isoforms during embryogenesis. J Cell Biol 1991;112:355-62.

9 Pfander D, Cramer T, Deuerling D, Weseloh G, Swoboda B. Expression of Thrombospondin-1 and its receptor CD36 in human osteoarthritic cartilage. Ann Rheum Dis 2000:59:448-54.

10 Aigner T, Vornehm SI, Zeiler G, Dudhia J, von der Mark K, Bayliss MT. Suppression of cartilage matrix gene expression in upper zone chondrocytes of osteoarthritic cartilage. Arthritis Rheum 1997;40:562-9.

11 Vignon E, Balblanc JC, Mathieu P, Louisot P, Richard M. Metalloprotease activity, phospholipase A2 activity and cytokine concentration in osteoarthritis synovial fluids. Osteoarthritis Cartilage 1993;1:115-20.

12 Smith MD, Triantafillou S, Parker A, Youssef PP, Coleman M. Synovial membrane inflammation and cytokine production in patients with early osteoarthritis. J Rheumatol 1997:24:365-71.

13 Tetlow LC, Adlam DJ, Wooley DE. Matrix metalloproteinase and proinflammatory cytokine production by chondrocytes of human osteoarthritic cartilage: associations with degenerative changes. Arthritis Rheum 2001;44:585-94.

14 Moos V, Fickert S, Müller B, Weber U, Sieper J. Immunohistological analysis of cytokine expression in human osteoarthritic and healthy cartilage. J Rheumatol 1999;26:870-9.

15 Martel-Pelletier J, Di-Battista J, Lajaneusse D. Biochemical factors in joint articular tissue degradation in osteoarthritis. In: Reginster JY, Pelletier JP, Martel-Pelletier J, Henroitin Y, eds. Berlin, New York, Heidelberg: Springer, 1999:156-87.

16 Goldring MB, Birkhead J, Sandell U, Kimura T, Krane SM. Interleukin-1 suppresses expression of cartilage-specific types II and IX collagens and increases types I and III collagens in human chondrocytes. J Clin Invest 1988:82:2026-37.

17 Stöve J, Huch K, Günther KP, Scharf HP. Interleukin-1 beta induces different gene expression of stromelysin, aggrecan and tumor necrosis factor stimulated gene 6 in human osteoarthritic chondrocytes in vitro. Pathobiology 2000;68:144-9.

18 Von der Mark K, Kirsch T, Nerlich A, Kuss A, Weseloh G, Glückert K, et al. Type $X$ collagen synthesis in human osteoarthritic cartilage. Arthritis Rheum 1992;35:806-11.

19 Pullig O, Weseloh G, Ronneberger DL, Käkönen SM, Swoboda B. Chondrocyte differentiation in human osteoarthritic cartilage: expression of osteocalcin in normal and osteoarthritic cartilage and bone. Calcif Tissue Int 2000;67:230-40.

20 Pfander D, Swoboda B, Kirsch T. Expression of early and late differentiation markers (PCNA, syndecan-3, annexin VI and alkaline phosphatase) by human osteoarthritic chondrocytes. Am J Pathol 2001;159:1777-83.

21 Salter DM. Tenascin is increased in cartilage and synovium from arthritic knees. Br J Rheumatol 1993;32:780-6.

22 Chevalier X, Groult N, Larget-Piet B, Zari L, Hornebeck W. Tenascin distribution in articular cartilage from normal subjects and from patients with osteoarthritis and rheumatoid arthritis. Arthritis Rheum 1994;37:1013-22.

23 Mankin H, Dorfman H, Lippiello L, Zarins A. Biochemical and metabolic abnormalities in articular cartilage from osteoarthritic human hips. II. Correlation of morphology with biochemical and metabolic data. J Bone Joint Surg Am 1971;53:523-37.

24 Noda N, Minoura H, Nishiura R, Toyoda N, Imanaka-Yoshida K, Sakura T, et al. Expression of tenascin-C in stromal cells of the murine uterus during early pregnancy: induction by interleukin-alpha, prostaglandin $E(2)$, and prostaglandin F(2alpha). Biol Reprod 2000;63:1713-20.

25 Chevalier X, Claudepierre P, Groult N, Godeau GJ. Influence of interleukin $1 \beta$ on tenascin distribution in human normal and osteoarthritic cartilage: a quantitative immunohistochemical study. Ann Rheum Dis 1996;55:772-5.

26 Aigner T, Zien A, Gehrsitz A, Gebhard PM, McKenna L. Anabolic and catabolic gene expression pattern analysis in normal versus osteoarthritic cartilage using DNA-array technology. Arthritis Rheum 2001;44:2777-89.

27 Smith RL, Lin J, Trindade MC, Shida J, Kajiyama G, Vu T, et al. Timedependent effects of intermittent hydrostatic pressure on articular chondrocyte type II collagen and aggrecan mRNA expression. J Rehabil Res Dev 2000;37:153-61.

28 Pfander D, Cramer T, Schipani E, Johnson RS. HIF- lalpha controls extracellular matrix synthesis by epiphyseal chondrocytes. J Cell Sci 2003;116:1819-26. 